\title{
Rhinology Future Debates 2018, a EUFOREA Report *
}

Emmanuel Prokopakis', Alexios Vardouniotis², Claus Bachert³, Jean Bousquet ${ }^{4}$, Sean Carrie ${ }^{5}$, Paolo Castelnuevo ${ }^{6}$, Jannis Constantinidis ${ }^{7}$, Wytske Fokkens ${ }^{8}$, Claire Hopkins ${ }^{9}$, Andreas Leunig ${ }^{10}$, Gabriele Molteni ${ }^{11}$, Joaquim Mullol'12, Glenis Scadding ${ }^{13}$, Sven Seys ${ }^{14}$, Gert Marien ${ }^{14}$, Maria Doulaptsi', Peter Hellings $3,7,15$

'Department of Otorhinolaryngology-Head and Neck Surgery, University of Crete School of Medicine, Heraklion, Crete, Greece 2 ENT Department, St Mary's Treatment Centre, Portsmouth, UK

${ }^{3}$ Ghent University Hospital, Ghent, Belgium

${ }^{4}$ University Hospital, Montpellier, MACVIA-France, Fondation partenariale FMC VIA-LR, Montpellier, France

5 ENT Department, Newcastle upon Tyne NHS Hospitals FT, Newcastle upon Tyne, UK

${ }^{6}$ Department of Otorhinolaryngology, University of Insubria and ASST Sette Laghi, Ospedale di Circolo, Varese, Italy

${ }^{7}$ 1st Department of Otorhinolaryngology, Head and Neck Surgery, AHEPA Hospital, Aristotle University of Thessaloniki, Greece

${ }^{8}$ Department of Otorhinolaryngology, Academic Medical Centre, Amsterdam, The Netherlands

'ENT Department, Guy's and St Thomas' Hospital, London, UK

${ }^{10}$ Rhinology Center, ENT-Clinic Bogenhausen, Dr. Gaertner GmbH, Munich, Germany

"Otorhinolaryngology-Head and Neck Surgery Department, University Hospital of Verona, Verona, Italy

${ }^{12}$ Clinical and Experimental Respiratory Immunoallergy, Hospital Clínic, IDIBAPS, Barcelona, Spain

${ }^{13}$ The Royal National Throat Nose \& Ear Hospital, London, UK

${ }^{14}$ European Forum for Research and Education in allergy and Airway Diseases (EUFOREA), Brussels, Belgium

${ }^{15}$ Clinical Division of Otorhinolaryngology, Head and Neck Surgery, University Hospitals Leuven, Leuven, Belgium
Rhinology 58: 0, 0 - 0, 2020

https://doi.org/10.4193/Rhin19.455

*Received for publication:

December 16, 2019

Accepted: January 26, 2020

\section{Abstract}

The third Rhinology Future Debates was organized by the European Forum for Research and Education in Allergy and Airways diseases (EUFOREA) in 2018 in Brussels. Experts from different specialties and countries, alongside patients, health policy makers and industry representatives discussed relevant topics in Rhinology, in an attempt to improve current clinical practices, through implementation of precision medicine, by empowering patients' participation and the use of eHealth tools. The debates which are available on-line (www.rhinology-future.com) dealt with 5 topics in Rhinology: the adoption of allergen-specific immunotherapy (AIT) by implementing change management strategies, the needs and obstacles in care delivery in respiratory diseases, 3D technology in nose and sinus surgery, ambulatory nasal surgery, and clinical evidence for efficacy of biologicals in CRSwNP and asthma.

This report summarizes the outcomes of the brainstorming sessions highlighting novel approaches and unmet needs in the field of respiratory diseases by focusing on integrated care pathways.

Key words: EUFOREA, debates, AIT, biologicals, CRSwNP, asthma, 3D, integrated care pathways

\section{Introduction}

The third Rhinology Future Debates was held in Brussels in November 2018, organized by EUFOREA (European Forum for Research and Education in Allergy and Airways diseases, www. euforea.eu).
For the first time in Rhinology, a peer to peer scientific exchange of expert opinions from the fields of rhinology, respiratory care and health policy with the participation of patients led to brainstorming and discussion on a number of issues in Rhinology. Novel developments were presented by key opinion leaders 
in Allergology and Rhinology, patients added their personal beliefs, then followed lively debate on the potential positioning of new developments in care pathways, the strengths and weaknesses of novel developments ${ }^{(1,2)}$.

The 5 topics for the 2018 debates were the following:

1. How to smooth the adoption of allergen-specific immunotherapy (AIT) by implementing change management strategies.

2. The needs and obstacles in care delivery in respiratory diseases.

3. $3 \mathrm{D}$ technology in nose and sinus surgery.

4. Ambulatory nasal surgery.

5. Clinical evidence of efficacy of biologicals in CRSwNP and asthma.

As all debates are recorded and distributed on-line with limited editing (www.rhinology-future.com), EUFOREA aims at maximizing the education of the target groups on novel developments, allowing critical appraisal of the future and more rapid implementation of promising novel tools, techniques and/or molecules in clinical practice in Europe ${ }^{(3)}$.

\section{How to smooth the adoption of AIT by implemen- ting change management strategies in the Rhino- logy clinic}

The prevalence of allergic rhinitis (AR) reaches up to $20-25 \%$ in the EU, with up to $30-35 \%$ of these subjects being uncontrolled in spite of available and recommended pharmacotherapy ${ }^{(4,5)}$. It is likely that $10 \%$ of the total AR patient population is suitable for AIT ${ }^{(6,7)}$. AR costs the EU 50 to 100 billion Euros annually, indirect cost almost doubling direct cost ${ }^{(3,8)}$. Delay in access to effective treatments leads to large numbers of uncontrolled patients and increases the disease's socio-economic impact. In order to gradually reduce the pool of uncontrolled patients one needs to consider methods for better adoption of AIT for AR and allergic asthma ${ }^{(9)}$. AIT with specific products and protocols is proven to be effective in selected AR patients; with reduction of AR symptoms and the need for rescue medications and improvement in quality of life. Moreover AIT provides clinical benefit for years after treatment discontinuation as immune tolerance for allergen(s) is induced and importantly AIT can probably reduce progression of $A R$ to asthma, thus providing major cost savings ${ }^{(10-15)}$.

Regarding the economics of AIT, only some countries reimburse such treatments while others do not. More health technology assessment studies are needed to ensure that efficacy is associated to cost effectiveness and translated to reimbursement. In order to achieve integration to health care practices there are four important areas: prevention, diagnosis, treatment and follow up. Prevention involves timely access to treatment, for example giving AIT to AR patients. This involves education of doctors and raising patient awareness by the use of novel digital tools such as allergic diary apps and AR on-line platforms. The second step should be the simplification of guidelines including real-world data in order to increase their implementation in real life. Education of all health care providers is crucial to achieve more access to AIT. Furthermore monitoring AR patients could boost AIT result data substantiating the benefits of treatment and its cost effectiveness. Finally consideration of patients' needs to ensure a smooth sequence of care and delivery steps is necessary. This might involve a multidisciplinary integrated tracking care pathway.

In general, all panel members acknowledged the efficacy of AIT, as proven by randomized controlled trials (RCT) and real-life studies. However, there were remarks regarding studies included in the guidelines since RCTs are used for the registration of products but do not reflect real life ${ }^{(7,16-18)}$. Some of the panelists pointed out that guidelines are based on ideal patients who show high compliance in trials and particular products, hence requiring careful interpretation.

A new era is emerging called the "Next generation care pathways for Rhinitis and Asthma - a model for non-communicable diseases" (19-21), and real life studies will be performed with the use of digital tools (cell phones and online platforms) in order to check if the proposed recommendations actually fit into real life (22-26).

Additionally, pharmacy-economic AIT studies should be based on real world evidence including direct medical costs and indirect costs such as improvement of school and work productivity, therefore justifying the need for reimbursement. Reimbursement should be for documented products and not for those without proven evidence (27).

Regarding education, all panelists agreed on the crucial need to educate all stakeholders including patients, physicians and politicians on the indication and outcomes of AIT. New ways of education are essential in every chronic disease, possibly as in medically focused internet search engines for educating patients. It was proposed that AIT should be a part of the basic training during medical school in order to raise awareness in the medical community. Moreover, AIT should be transferred to the hands of a multidisciplinary team instead of one individual and specialty.

The panelists concluded that there should be better communication, feedback and interaction between AR patients and physicians from different specialties dealing with AR.

Patients have identified a lack of knowledge regarding the right treatment and medication by the general practitioners (GPs) for decades, depriving patients from the therapeutic option of AIT (Figure 1) (21).

At this point all participants agreed that we need to agree how to maximize digital platform usage, but at the same time gua- 


\section{EUFOREA}

4 Key Barriers / Solutions throughout the holistic care continuum Efficiency and Multidisciplinary Collaboration

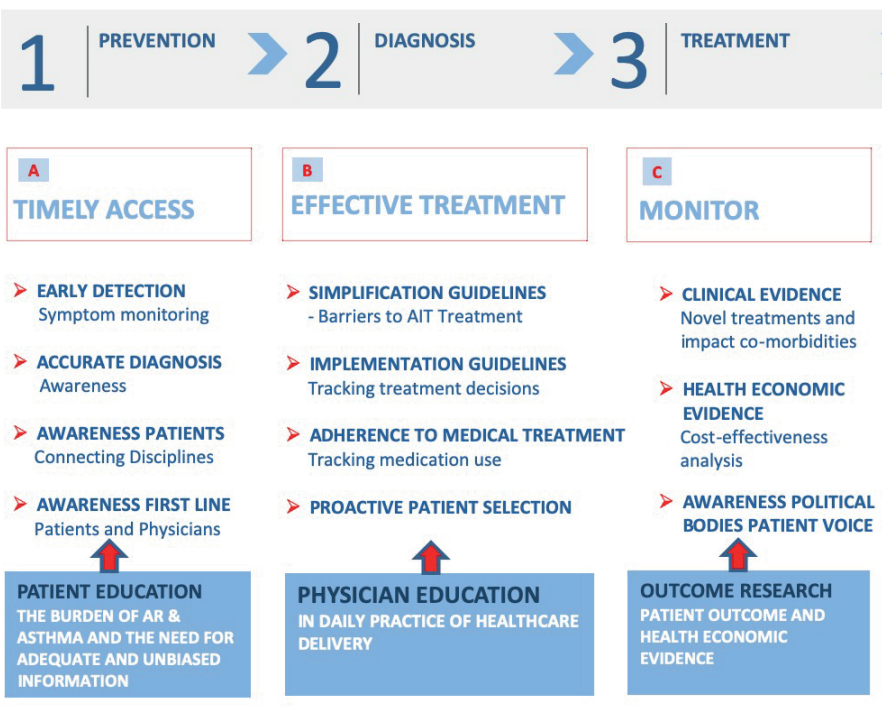

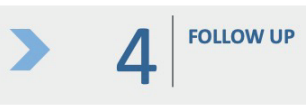

D EFFICIENCY + COLLABORATION

$>$ FACILITATION NECESSARY VISITS Patient behaviour

$\checkmark$ SMOOTH SEQUENCE Of CARE DELIVERY STEPS Tracking Care Pathway

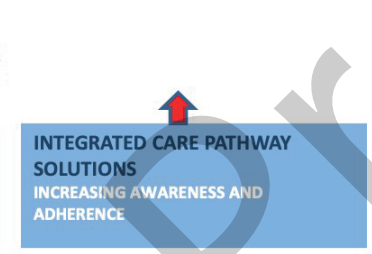

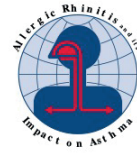

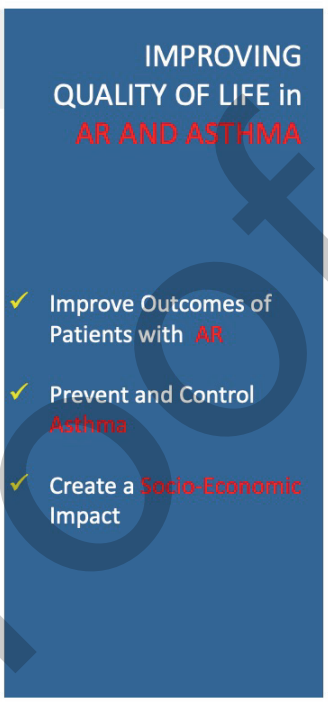

Figure 1. The four-step approach from EUFOREA for improving quality of life in CRDs.

rantee information security and avoid the spread of unverified recommendations. There should be an agreement or a partnership of scientific societies under the lead of EUFOREA working with patients' organizations in proposing the correct education. The need for continuous remote patient monitoring (with apps) and the deployment of an outcome registry for research purposes was highlighted. We must be open to the idea of creating new infrastructures between the patients and the physicians in order to increase adherence, since this is the key to success in terms of efficacy. However, there are many problems to consider due to the privacy rules of EU. For example a messaging system to patients was proposed as a solution to possibly help adherence and treatment, but the app would need to conform to CE directives as a Class IIA medical device.

The conclusion of the debate was that first the evidence from RCTs is needed, then real life studies and integrated care pathways utilizing digital tools. Patient and physician education is vital and all must work collectively, be open-minded and innovative to face the challenges.

Many different systems can be used. As an example, EUFOREA may take on the challenges related to AIT adoption in real-life, and is launching the Galenus Health system for education of patients and physicians, patient follow-up and outcome registry.

\section{Take home messages:}

- $\quad$ AIT using standardized allergens and validated protocols is highly effective in AR patients.

- Education of doctors and raising patient awareness by the use of novel digital tools (allergic diary apps and AR on-line platforms) is crucial for dealing with AR, and asthma prevention.

Digital tools will help to reinforce real world studies and integrated care pathways.

Reimbursement should be applied only for documented products, not for those without proven evidence using heath technology assessment.

\section{The needs and obstacles in care delivery in respi- ratory diseases}

The overview of the discussion was that there are 3 winning moves (Figure 2) regarding better control of patient outcomes in Chronic Respiratory Diseases (CRD's) including:

1. The design and implementation of integrated solutions concerning prevention, diagnosis, treatment and follow-up for all stakeholders through holistic care pathways.

2. The leverage of digital capabilities required to support the integrated solutions, digitally enabling patient centric care.

3. Continuously implementing these integrated digital solutions to accelerate changes in daily clinical practice.

In particular, the need forpatient educational programs was noted in order to increase the patient awareness and that of first line care health care providers, enabling early detection and more adequate diagnosis at the GP level. In addition, physician educational programs are necessary to support better implementation of guidelines into practice and better adherence to medications for patients, eventually increasing treatment 


\section{Winning moves}

EUFOREA to better control outcomes in chronic respiratory diseases

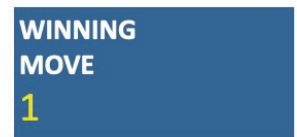

\section{WINNING}

MOVE

2

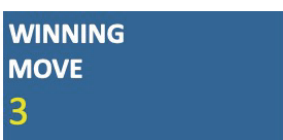

DESIGN INTEGRATED SOLUTIONS

FOR ALL STAKEHOLDERS THROUGH THE HOLISTIC CARE PATHWAY

LEVERAGE DIGITAL CAPABILITIES

TO FACILITATE DIGITALLY-ENABLE AND PATIENT CENTRIC CARE

IMPLEMENT RELENTLESSLY AND COURAGEOUSLY

TO ACCELERATE CHANGE IN DAILY PRACTICE

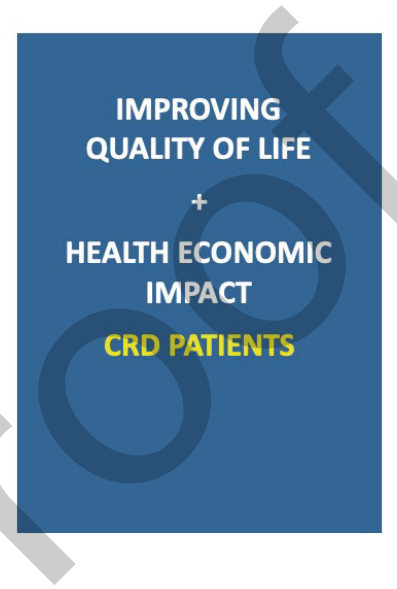

Figure 2. Winning move for better control of patient outcomes in CRDs.

effectiveness. Furthermore, the need for outcomes registries was underlined in order to collect data - both clinical and economical-for more research and exchange of best practices amongst physicians. Finally, the need to deploy integrated care pathways was stated, to facilitate collaboration between primary, secondary and tertiary levels of care, enabling patient exposure to the right treatment, at the right time and the right location.

The aforementioned procedure can be made simpler through digital solutions such as e-learning educational platforms for patients and physicians, digital apps, outcome registries, physician dashboards or clinical decision support systems ${ }^{(25,28)}$. The panel agreed that we need to collaborate in order to leverage all our capabilities and create synergies, overcoming implementation barriers - such as technological constraints and legal compliance that relates to General Data Protection Regulation (GDPR). Last but not least, we need to overcome resistance to change by mobilizing patients' commitment - creating traction and accelerating change in daily practice.

From the patients' perspective of real-life care delivery for chronic rhinosinusitis (CRS), it was mentioned that digital tools as in smart phone applications (mySinusCoach, Allergy Diary, MASK AIR) and platforms can be helpful. Patients are currently struggling to find accurate and up-to-date information ${ }^{(29,30)}$. Additionally, the question of how to involve the patient in the process of early diagnosis, treatment and follow-up of disease still needs to be addressed, as well as the need for more detailed and specific information for the experienced patient. Probably the most unprecedented request was that of a system that could alert or detect an upcoming loss in controlling symptoms. Finally the patients seem overwhelmingly willing to have their data anonymously utilized in a research environment under a collective effort in order to create better treatment protocols ${ }^{(31)}$.

As a response the panel of experts agreed that at the centre of any digital solution is end- user involvement in the development process, changing the way we practice medicine to a patient centered approach. The patient's need to engage in a simple and effective way was acknowledged as was the need to reassure each patient that the information they input is actually seen by a healthcare practitioner. It was proposed that the data from the apps need to be assessed from the patients' point of view as well as that of physicians, since the results involve mechanisms that differ in different patients and adherence to treatment that is extremely low ${ }^{(21)}$.

The panelists discussed the digital transformation of health aiming for a patient- centered approach in order to change the management of chronic diseases, since there is an enormous gap between what is taught in medical schools, what we practice and what the patient does. Regular long-term treatment is associated with extremely poor adherence ${ }^{(16,32)}$. Furthermore the necessity for change to the mentality of physicians to make the patient an active partner of the decision making progress, leading to real life implementation of precision medicine, higher adherence, improved level of control, and enhanced cost-effectiveness ${ }^{(12,33)}$.

The key aspect was that the digital system may allow patients' concerns, physician prescribing and all information to be integrated in a manner that could be "tailored" to the patient. Education in medical schools also needs to be reshaped to a 
holistic approach, since currently it is done through a physician's perspective. Digital platforms will allow us to move from a static moment in time regarding patient's symptoms and history to a real life environment over the course of months prior to consultation ${ }^{(2,34)}$. The procedure should be time saving for doctors since they will be able to refer patients to a site or app were they can find certified information matching those of the applied guidelines and the treating physician.

Digital tools can reduce the level of confusion amongst patients getting conflicting information from the pharmacist, primary care physicians, and specialists from tertiary care level providers. The apps are not a solution on their own, but are part of a system working to support integrated care pathways through a personalized treatment plan, that the patient will choose to follow or not, moving from one step to another, over a period of time. The physician will be able to follow the patient remotely, 24 hours a day if needed, adjusting the time and date of a visit as a priority if there is poor control.

It was sensible that such a debate had a specialist to comment on GDPR. It was mentioned that all the rules regarding data protection need to be applied from the very beginning, since that is the start of the chain and anything used from that point and beyond needs to be compliant withregulations. Additionally, transparency of the obtained data is necessary, which means that one needs to state what kind of data you will be using in a way that the data provider can understand. Furthermore, data minimization is important, only collecting the data that you will be analyzing and not more. Equally vital is the "principle of purpose": data can only be used for the specific reason it was collected and the patient understands the purpose of the data processing. The aforementioned parameters cannot be covered by anonymous data. Patient consent is necessary and in particular a "broad consent" where the patient agrees to share the data, but could revoke the decision at any time. Furthermore it was mentioned that once people understand the purposes which their data will be used for they provide it more willingly ${ }^{(35,36)}$. Nevertheless, it was pointed out that legal obligations through GDPR should not be considered as a barrier but an opportunity, since strong legislation only means that the patient is aware and actually willing to share the data added to stronger rights. The opinion of the panel was that in the future it will be probably common to give treatment advice to the patients through apps and digital solutions.

Finally, the discussion included possible barriers regarding the implementation of user- friendly digital tools that are fine tuned and personalized. Additionally all health care providers need to be convinced into recommending e-health tools as a support. The challenge for physicians is the development of appropriate tools, their dissemination and finally merging into one integrated solution that will be superior to the current health system in Europe. It was acknowledged however that the use of techno- logy could not possibly be the solution for everybody and that technological solutions have not been proven to be economically beneficial. Therefore more studies are required to empower governments to provide an arena for implementing technological solutions ${ }^{(3,20,37)}$.

The conclusion of the debate was that multiple solutions are needed to develop better outcomes for patients with CRDs in the fields of patient education, physician education, registries and pathways and that digital methods play a crucial role to support the deployment of these solutions. The EUFOREA team is encouraged to take the lead into the development of a novel e-health tool that will reduce the obstacles for optimal care in CRD $(2,12,31)$.

Take home messages:

- Patient educational programs are necessary in order to increase the awareness of patients and first line care health care providers, enabling early detection and more adequate diagnosis of CRD.

- Friendly digital tools that are fine tuned and personalized could be used to give treatment advice in CRD.

- GDPR should be considered as an opportunity for better data utilization and processing.

The EUFOREA team could lead the development of a novel e-health tool that will reduce the obstacles for optimal care in CRD.

\section{3D technology in nose and sinus surgery}

The third part of the debate was about the use of the VITOM ${ }^{\oplus}$ 3D with TIPCAM ${ }^{\oplus} 1$ S 3D ORL by Storz in sinus and skull base surgery. Initially, various 3D technologies were used to recreate an artificial three dimensional environment. The VITOM 3D consists of an exoscope with 2 cameras at the tip, therefore not providing a reconstructed image but a true high definition stereoscopic vision that can be used in the vast majority of the ENT surgical field. The system moves from the classic "tunnel vision" to a more broadened vision, by including peripheral landmarks. Additionally it occupies less space in the operating room compared to the classic setup when using a microscope and with the use of a new $45^{\circ}$ scope no changing of scopes (from $0^{\circ}$ or $30^{\circ}$ ) during surgery is required. The aforementioned data presenta breakthrough in nasal and skull base surgery and therefore the group of experts was called on to discuss the advantages and disadvantages of the system and the use of $3 D$ technology in general.

Some of the members of the panel referred to 3D technology as a revolution due to the excellent visualization of the surgical field in a stereoscopic vision mainly in open procedures and that only the microscope used to provide. The main advantage of 3D vision is the ability to better assess the depth of the anatomical structures, added to excellent magnification and reproduction 
of colors. Additionally most of the expert surgeons agreed that it is an excellent tool for educational purposes, when it comes to teaching residents and fellows. It was mentioned through the literature that surgery with the use of 3D technology has been proven to be even less invasive and reduces surgical time, therefore being beneficiary for the patients as well by shortening recovery time. From a technical point of view all the members agreed that the system functions better in a wider space - as the posterior sinuses, the sphenoid sinus or the skull base - compared to narrow spaces - as the anterior part of the nasal cavity - therefore raising the issue of if it has to be used in each and every patient undergoing sinus surgery. The 3D technology fits fully in the emerging field of Rhinology and is especially helpful in advanced trans cranial surgery where every potential maneuver is life threatening. On the other hand everybody noted that bleeding can be an issue, since if one of the two lenses is covered then the vision you have is no longer $3 \mathrm{D}$ but $2 \mathrm{D}$ and therefore the control of bleeding is vital for the uncomplicated use of the system. As in any surgical procedure there is a learning curve that needs to be achieved. Interestingly enough it was mentioned that a less experienced surgeon could take on the whole concept much more easily.

Regarding the issue of hospitals investing in this kind of technology several opinions were put on the table. Since complications and morbidity rate in endonasal procedures and sinus surgery are already low, some of the panelists stated that it is rather difficult to convince hospital managements to accept the challenge, whereas others believed that for an academical -training hospital - it is absolutely necessary and on that basis, denying the investment could be easier overpowered. The argument being that it allows the fellows to better comprehend the anatomy and the steps of sinus surgery. One panelist made a remark in regards to the recording and the projecting images of the system that could be further improved in the future, making the technology even more appealing.

Furthermore, there were no arguments against applications of $3 \mathrm{D}$ technology as in 3D morphing - explaining to the patients of the anticipated outcomes of surgery - and 3D printing - scaffolds and tissue engineering- in the field of rhinoplasty ${ }^{(38-41)}$. Such technology is currently in use by several academic centers around Europe, since as it was said it is the "way to the future" fitting in perfectly with the concept of novel trends in Rhinology (42).

In conclusion, a few panelists were still skeptical if this is the end of the line in technology evolution for the time being, making 3D systems a "must" for each and every hospital, or if a period of watchful waiting in needed to see what the future brings. The majority of the group, however, expressed the belief that 3D technology in sinus and skull base surgery is exactly what we have been awaiting for a very long time.
Take home messages:

- 3D technology can be an excellent tool for educational purposes, so as to teaching residents and fellows.

- As with the implementation of any technological tool, there is a learning curve that needs to be achieved.

- 3D technology in sinus and skull base surgery is the "way to the future" fitting in perfectly with the concept of novel trends in Rhinology.

\section{Ambulatory nasal surgery}

Ambulatory nasal surgery is clinic and not theatre based, is done under local rather than general anesthesia and usually there is no provision for sedation (local anesthetic outpatient nasal surgery) ${ }^{(43)}$. However, it was mentioned from the panelists that different models exist in their countries, including sedation, varying in the surgical procedures that are performed - expanding from septoplasty and sub mucosal diathermy of the lower turbinates (reduction), to polypectomy and limited FESS or dacryocystorhinostomy- to the venue where the procedure takes place (day care surgery, in hospital or private practice/clinic) (44-46). The "driver" behind ambulatory interventions is mainly the "patient's choice", since patients are nowadays more aware that nasal surgery can be done under local anesthesia and therefore expressing a preference for less invasive operations. For the clinicians, culture of innovation and new technologies regarding medical equipment (microdebriders, pediatric endoscopes) and growing experience in the field are the main reasons to consider ambulatory treatment. For the healthcare system, cutting down the large waiting lists and reducing the cost compared to major surgery are the basic incentives ${ }^{(47-50)}$. Even though we lack large studies to compare between the results of surgery performed in a standard surgical setting and those when performing ambulatory nasal surgery, the data are encouraging and supportive, in means of reducing time, accomplishing the same level of patient satisfaction and having milder side-effects.

The patient representative was concerned regarding the physical experience and efficacy of such a procedure, after commenting that convenience is definitely a factor for actually selecting it over major surgery under general anesthesia.

The key factor of success of ambulatory surgery is the proper selection of patients based on the patient's and surgeon's preferences and experience, as well as patient's personality and need for excellent collaboration. Even though patients without accompanying comorbidities are considered the best candidates for such a treatment, in many cases, it is possible that even patients and comorbidities could be considered for such a procedure, due to contraindications toundergoing general anesthesia. It was stated by one of the panelists that having the ability to switch to general anesthesia with the assistance of an anesthesiologist if necessary, could further increase the cases were such procedures to be performed. Patients with 
cardiopulmonary diseases, or under anti-coagulant therapy, or with a history of DVT (Deep Vein Thrombosis), as well as older patients are quite difficult to be managed in ambulatory nasal surgery. Additionally, it was reported that even healthy patients can present intraoperative complications, especially when the duration of the procedure exceeds 30' minutes. Obviously, major surgery for extended disease (Draf III, nasal malignancies) cannot be done under any kind of local anesthesia in an outpatient environment.

Regarding health economics and assessing if ambulatory nasal surgery is equivalent or superior compared to standard surgical procedures, the discussion was even more complicated. Initially it was stated that the lack of data cannot provide a crystal clear answer to that debate, warranting the need for more studies regarding cost and effectiveness of ambulatory nasal surgery. Unfortunately, most of the evidence at this point is based on personal opinions and experience, without having comparative data on clinical outputs and true benefits.

The concept of value based healthcare is a fraction of the quality of the clinical outcome divided by cost, with both being more difficult than thought to calculate. . The total cost of a surgical procedure as in the whole cost, expanding outside the hospital back to caretakers, family, etc. is much different. Quality of the outcome is even more difficult to define and measure, even without contemplating subjective preference, since it involves the satisfaction of the patient withthe quality of the provided services, comfort and well-being, convenience and safety, to the satisfaction of the surgeon having performed a successful operation.

Finally, regarding t the venue where ambulatory nasal surgery can be performed, all of the panelists agreed that the patient and the physician mainly need to decide upon the actual treatment rather than having an available setting offer a controversial therapy ${ }^{(50-52)}$.

The conclusion of the discussion was that there are challenges regarding patient selection and the venue of the procedure in ambulatory nasal surgery, however, undoubtedly the panel agreed that safety should be the main objective in each and every case, probably by working within a safe environment that is linked to a hospital. Nevertheless the main argument that still needs to be addressed is health economics - cost efficacy - that is a very variable factor across the different countries.

Take home messages:

- Increasing demand for services and limited resources make the concept of ambulatory nasal surgery appealing again.

- Patients' selection for ambulatory nasal surgery should be a multifactorial process that needs to be based on the patient's wishes and the surgeons training, within the frame of performing evidence based surgery and not compromising the surgical plan.
- Data for ambulatory nasal surgery are encouraging and supportive, in means of reducing time, accomplishing the same level of patient satisfaction and having milder sideeffects ${ }^{(48)}$.

\section{Clinical evidence of biological in CRSwNP and Asthma}

Biological treatments have been used successfully in the treatment of severe asthma (Omalizumab, Mepolizumab, Benralizumab, Reslizumab and Dupilumab) ${ }^{(53)}$. Chronic rhinosinusitis with nasal polyps (CRSwNP) is also considered a chronic respiratory diseases, often associated with asthma, in which monoclonal antibodies may be introduced in the near future ${ }^{(52,54-58)}$. Studies have shown that biological drugs are effective in asthma in reducing exacerbations, improving lung function, reducing oral corticosteroid use and improving quality of life. Approximately $70 \%$ of patients with CRSWNP have recurrent disease over time, especially those with comorbid asthma and/or NSAIDexacerbated respiratory disease ${ }^{(52)}$. A recent study showed the potent effect of dupilumab in CRSwNP ${ }^{(57,59)}$.

Endotyping CRS based on cluster analysis from the expression of specific cytokines in CRSwNP can help define those patients that might benefit from treatment with monoclonal antibodies ${ }^{(60)}$. In particular nasal polyp patients who present with a type-2 immune reaction - with high levels of IL-5, IgE ECP all in full first time, complicated by the impact of Staphylococcus aureus locally (SE-IgE) and frequently by asthma are eligible for biological treatment, if uncontrolled by conventional therapy ${ }^{(58)}$. In one randomized clinical trial of 60 patients subcutaneous dupilumab after 4 months significantly improved the endonasal NPS (Nasal Polyp Score) by 2 points, and improved the SNOT 22 quality of life score in CRSwNP (61,62).

Based on well-conducted trials, indications of the level of efficacy of biologicals for CRSwNP and of the means to identify likely responders will soon be available. At the time of the debate in 2018, there were 4 phase III clinical studies in progress. It was highlighted in the debate that targeting the "united airway concept" by improving both upper and lower airways outcomes simultaneously by monoclonal antibodies need to be performed. Monitoring organizations such as the FDA (Food and Drug Administration), which insist on trial programs of either asthma or of nasal polyposis first, do currently not support this combined approach.

Reimbursement formed a large part of the debate. There are multiple considerations when recommending surgery, revision surgery or the use of monoclonal antibodies to a patient, linked to efficacy, cost, safety, comparison and duration of efficacy, etc. It was stated that reimbursement cannot be based strictly on biomarkers since biomarker levels in nasal polyps are lower than those seen in asthma, as the eosinophils may not increase in the blood and IgE may not increase in the serum. On the other 
hand it was stated without doubt that patient with a late onset asthma and nasal polyps is almost $95 \%$ certain to be a patient with Type 2 inflammation that could definitely benefit from biological treatment ${ }^{(56)}$. The health authorities' representative underlined the need for more phase III clinical trials and studies with robust data to prove the effectiveness of such medications in CRSwNP, after which the relevant pharmaceutical company needs to obtain authorization from EMA (European Medicine Agency) prior to requesting reimbursement. The evaluation leading to the decision- making process will include benefit-risk evaluation versus placebo or standard care, including efficacy, safety and cost compared to existing therapies. In the UK the regulatory body - NICE - has what is called a "ceiling price" referring to a maximum amount of money that can be spent on any new drug, therefore framing the discussion for the negotiations between the price the drug company is asking for and the criteria for the number of patients receiving the treatment as indicated by physicians. It also looks at cost per QALY - the amount needed to improve quality of life by 1 unit. Everybody agreed that the patients' voice should enter into the discussion, a fact that received enthusiastic acceptance from the patients' perspective as well ${ }^{(31,37)}$. EUFOREA has already introduced a digital tool (MySinusitisCoach) to broaden the patients' awareness regarding CRSwNP, treatment options and therapeutic strategies, including expectations after surgery ${ }^{(29)}$. In due time, when biologicals find their position in treatment, then the patients can be educated accordingly.

Furthermore integrated care pathways can contribute to the adoption of biologicals through developing a certain algorithm of treatment and streamlining movement along the pathway ${ }^{(32,63)}$. It was proposed that better educating primary care physicians to select these patients and promptly offer the appropriate treatment would save time and money ${ }^{(50,51,64,65)}$. Moreover ENT doctors need to better recognize those patients who will not benefit from a single dose of oral steroids, a simple polypectomy or a limited FESS procedure and have them referred to an Academic Center to have a more extended and efficient procedure performed ${ }^{(33,34,66-70)}$.

\section{Conclusion}

In conclusion, more clinical studies are necessary to establish the cost effectiveness and efficacy of monoclonal antibodies in CRSwNP. The panel referred to biologicals as a possible "game changer" for a lot of patients - a fact that was acknowledged by the patients' representatives.

\section{Take home messages:}

- The EUFOREA expert panel defined criteria for the use of biological treatment in CRSwNP.

- More phase III clinical trials and studies with robust data will be performed to prove the effectiveness of biologicals in CRSwNP, so pharmaceutical companies can receive authorization from EMA prior to requesting reimbursement for the treatment.

- $\quad$ ENT doctors need to better recognize those patients who will not benefit from mainstream treatment protocols.

- Biologicals are considered "game changers" for physicians and patients in the field of Rhinology, particularly for patients with severe disease of the upper and lower respiratory tract.

\section{Acknowledgements}

None

\section{Authorship contribution}

$E P$ and $\mathrm{PH}$ contributed to conception and design of the study. AV, CB, JB, SC, PC, JC, WF, CH, AL, GM, JM, GS, SS, GM, and MD contributed to data analysis and interpretation.

$\mathrm{EP}, \mathrm{PH}, \mathrm{AV}, \mathrm{CB}, \mathrm{JB}, \mathrm{SC}, \mathrm{PC}, \mathrm{JC}, \mathrm{WF}, \mathrm{CH}, \mathrm{AL}, \mathrm{GM}, \mathrm{JM}, \mathrm{GS}, \mathrm{SS}, \mathrm{GM}$, and $M D$ participated in drafting the article and revising it critically. All authors gave final approval of the version to be submitted and any revised version.

\section{Conflict of interest}

The authors do not report any conflict of interest.

\section{References}

1. Ansa B, Goodman M, Ward K et al. Paranasal Fokkens WJ, Bachert C, Bernal-Sprekelsen M, Bousquet J, Djandji M, Dorenbaum A, et al. Rhinology Future Debates, an EUFOREA Report. Rhinology. 2017 Dec 1;55(4):298304.

2. Fokkens WJ, Pugin B, Bachert C, Bousquet J, Joos G, Louis R, et al. Rhinology Future Debates 2017 by EUFOREA: Novel treatments and surgical solutions in rhinology. Clin Otolaryngol 2018;43(6):1429-38.

3. Hellings PW, Borrelli D, Pietikainen S, Agache I, Akdis C, Bachert C, et al. European Summit on the Prevention and
Self-Management of Chronic Respiratory Diseases: report of the European Union Parliament Summit (29 March 2017). Clin Transl Allergy. 2017;7:49.

4. Brożek JL, Bousquet J, Agache I, Agarwal A Bachert C, Bosnic-Anticevich S, et al. Allergic Rhinitis and its Impact on Asthma (ARIA) guidelines-2016 revision. J Allergy Clin Immunol. 2017 Oct;140(4):950-8.

5. Hellings PW, Fokkens WJ, Akdis C, Bachert C Cingi C, Dietz de Loos D, et al. Uncontrolled allergic rhinitis and chronic rhinosinusitis: where do we stand today? Allergy. 2013 Jan;68(1):1-7.

6. Nurmatov U, Dhami S, Arasi S, Roberts G,
Pfaar O, Muraro A, et al. Allergen immunotherapy for allergic rhinoconjunctivitis: a systematic overview of systematic reviews. Clin Transl Allergy. 2017;7:24.

7. Bousquet J, Pfaar O, Togias A, Schünemann HJ, Ansotegui I, Papadopoulos NG, et al. 2019 ARIA Care pathways for allergen immunotherapy. Allergy. 2019; Nov,74 (11):2087-102.

8. Colás C, Brosa M, Antón E, Montoro J, Navarro A, Dordal MT, et al. Estimate of the total costs of allergic rhinitis in specialized care based on real-world data: the FERIN Study. Allergy. 2017 Jun;72(6):959-66.

9. Scadding $\mathrm{G}$, Bousquet J, Bachert C, Fokkens 
WJ, Hellings PW, Prokopakis E, et al. Rhinology future trends: 2017 EUFOREA debate on allergic rhinitis. Rhinology. 2019 Feb 1;57(1):49-56.

10. Devillier P, Wahn U, Zielen S, Heinrich J. Grass pollen sublingual immunotherapy tablets provide long-term relief of grass pollen-associated allergic rhinitis and reduce the risk of asthma: findings from a retrospective, real-world database subanalysis. Expert Rev Clin Immunol. 2017;13(12):1199206.

11. Zielen $S$, Devillier $P$, Heinrich J, Richter $H$, Wahn U. Sublingual immunotherapy provides long-term relief in allergic rhinitis and reduces the risk of asthma: A retrospective, real-world database analysis. Allergy. 2018 Jan;73(1):165-77.

12. Hellings PW, Fokkens WJ, Bachert C, Akdis CA, Bieber T, Agache I, et al. Positioning the principles of precision medicine in care pathways for allergic rhinitis and chronic rhinosinusitis - A EUFOREA-ARIA-EPOSAIRWAYS ICP statement. Allergy. 2017 Sep;72(9):1297-305.

13. Zuberbier T, Lötvall J, Simoens $S$, Subramanian SV, Church MK. Economic burden of inadequate management of allergic diseases in the European Union: a GA2LEN review. Allergy. 2014;69(10):1275-9.

14. Meadows A, Kaambwa B, Novielli N, Huissoon A, Fry-Smith A, Meads C, et al. A systematic review and economic evaluation of subcutaneous and sublingual allergen immunotherapy in adults and children with seasonal allergic rhinitis. Health Technol Assess Winch Engl. 2013 Jul;17(27):vi, xi-xiv, 1-322.

15. Asaria M, Dhami $S$, van Ree $R$, Gerth van Wijk R, Muraro A, Roberts G, et al. Health economic analysis of allergen immunotherapy for the management of allergic rhinitis, asthma, food allergy and venom allergy: A systematic overview. Allergy. 2018;73(2):269-83.

16. Hellings PW, Seys SF, Marien G, Agache I, Canonica W, Gevaert P, et al. ARIA masterclass 2018: From guidelines to real-life implementation. Rhinology. 2019 Oct 1;57(5):392-9.

17. Bousquet J, Arnavielhe S, Bedbrook A, Bewick M, Laune D, Mathieu-Dupas E, et al. MASK 2017: ARIA digitally-enabled, integrated, person-centred care for rhinitis and asthma multimorbidity using real-worldevidence. Clin Transł Allergy. 2018;8:45.

18. Bousquet J, Schünemann HJ, Togias $A$, Bachert C, Erhola M, Hellings PW, et al. Next-generation Allergic Rhinitis and Its Impact on Asthma (ARIA) guidelines for allergic rhinitis based on Grading of Recommendations Assessment, Development and Evaluation (GRADE) and real-world evidence. J Allergy Clin Immunol. 2019 Oct 15;

19. Bousquet JJ, Schünemann HJ, Togias $A$, Erhola M, Hellings PW, Zuberbier T, et al. Next-generation ARIA care pathways for rhinitis and asthma: a model for multimorbid chronic diseases. Clin Transl Allergy. 2019 Sep 9;9:44.

20. Agache I, Annesi-Maesano I, Bonertz A, Branca F, Cant A, Fras Z, et al. Prioritizing research challenges and funding for allergy and asthma and the need for translational research-The European Strategic Forum on Allergic Diseases. Allergy. 2019 Nov;74(11):2064-76.

21. Bousquet J, Bedbrook A, Czarlewski W, Onorato GL, Arnavielhe S, Laune D, et al. Guidance to 2018 good practice: ARIA digitally-enabled, integrated, person-centred care for rhinitis and asthma. Clin Transl Allergy. 2019;9:16.

22. Bousquet J, Ansotegui IJ, Anto JM, Arnavielhe S, Bachert C, Basagaña X, et al. Mobile Technology in Allergic Rhinitis: Evolution in Management or Revolution in Health and Care? J Allergy Clin Immunol Pract. 2019 Nov - Dec;7(8):2511-2523.

23. Maria Matricardi P, Dramburg S, AlvarezPerea A, Antolín-Amérigo D, Apfelbacher C, Atanaskovic-Markovic M, et al. The role of mobile health technologies in allergy care: An EAACI position paper. Allergy. 2020 Feb;75(2):259-272.

24. Bédard A, Basagaña X, Anto JM, GarciaAymerich J, Devillier P, Arnavielhe $S$, et al. Mobile technology offers novel insights into the control and treatment of allergic rhinitis: The MASK study. J Allergy Clin Immunol. 2019 Jul;144(1):135-143.e6.

25. Sleurs K, Seys SF, Bousquet J, Fokkens WJ, Gorris S, Pugin B, et al. Mobile health tools for the management of chronic respiratory diseases. Allergy. 2019 Jul;74(7):1292-306.

26. Menditto E, Costa E, Midão L, BosnicAnticevich S, Novellino E, Bialek S, et al. Adherence to treatment in allergic rhinitis using mobile technology. The MASK Study. Clin Exp Allergy J Br Soc Allergy Clin Immunol. 2019 Apr;49(4):442-60.

27. Bosnic-Anticevich $S$, Costa E, Menditto E, Lourenço O, Novellino E, Bialek S, et al. ARIA pharmacy 2018 'Allergic rhinitis care pathways for community pharmacy': AIRWAYS ICPS initiative (European Innovation Partnership on Active and Healthy Ageing, DG CONNECT and DG Santé) POLLAR (Impact of Air POLLution on Asthma and Rhinitis) GARD Demonstration project. Allergy. 2019 Jul;74(7):1219-36.

28. Seys SF, Quirce S, Agache I, Akdis CA, Alvaro-Lozano M, Antolín-Amérigo $D$, et al. Severe asthma: Entering an era of new concepts and emerging therapies: Highlights of the 4th international severe asthma forum, Madrid, 2018. Allergy. 2019 Nov;74(11):2244-8.

29. Seys SF, Bousquet J, Bachert C, Fokkens WJ, Agache I, Bernal-Sprekelsen $M$, et al. mySinusitisCoach: patient empowerment in chronic rhinosinusitis using mobile technology. Rhinology. 2018 Sep 1;56(3):209-15

30. Samreth D, Arnavielhe S, Ingenrieth F, Bedbrook A, Onorato GL, Murray R, et al. Geolocation with respect to personal privacy for the Allergy Diary app - a MASK study.
World Allergy Organ J. 2018;11(1):15

31. Pugin B, Deneyer L, Bachert C, Alobid I, Bousquet J, De Carlo G, et al. Patient Advisory Board for Chronic Rhinosinusitis A EUFOREA initiative. Rhinology. 2019 Oct 1;57(5):331-5

32. Fokkens W, Desrosiers M, Harvey R, Hopkins C, Mullol J, Philpott C, et al. EPOS2020: development strategy and goals for the latest European Position Paper on Rhinosinusitis. Rhinology. 2019 Jun 1;57(3):162-8.

33. Vlastos I, Gkouskou K, Doulaptsi M, Karatzanis A, Prokopakis EP. Precision Medicine in Rhinosinusitis. Curr Allergy Asthma Rep. 2019 Feb 22;19(2):12.

34. Hellings PW, Akdis CA, Bachert C, Bousquet J, Pugin B, Adriaensen G, et al. EUFOREA Rhinology Research Forum 2016: report of the brainstorming sessions on needs and priorities in rhinitis and rhinosinusitis. Rhinology. 2017 Sep 1;55(3):202-10.

35. Bousquet J, Arnavielhe S, Bedbrook A, Fonseca J, Morais Almeida M, Todo Bom A, et al. The Allergic Rhinitis and its Impact on Asthma (ARIA) score of allergic rhinitis using mobile technology correlates with quality of life: The MASK study. Allergy. 2018;73(2):505-10.

36. Bousquet J, Devillier P, Arnavielhe $S$, Bedbrook A, Alexis-Alexandre G, van Eerd $M$, et al. Treatment of allergic rhinitis using mobile technology with real-world data: The MASK observational pilot study. Allergy. 2018;73(9):1763-74.

37. Hellings PW. Joint action with European CRSwNP Patients for better outcomes. Rhinology. 2019 Oct 1;57(5):321.

38. Lekakis G, Hens G, Claes P, Hellings PW. Three-dimensional Morphing and Its Added Value in the Rhinoplasty Consult. Plast Reconstr Surg Glob Open. 2019 Jan;7(1):e2063.

39. Lekakis G, Claes P, Hamilton GS, Hellings PW. Three-Dimensional Surface Imaging and the Continuous Evolution of Preoperative and Postoperative Assessment in Rhinoplasty. Facial Plast Surg FPS. 2016 Feb;32(1):88-94.

40. Lekakis G, Claes P, Hamilton GS, Hellings PW. Evolution of Preoperative Rhinoplasty Consult by Computer Imaging. Facial Plast Surg FPS. 2016 Feb;32(1):80-7.

41. Prokopakis E, Doulaptsi M, Karatzanis A, Kawauchi H. Clinical Applications for Tissue Engineering in Rhinology. Turk Arch Otorhinolaryngol. 2019 Mar;57(1):39-41.

42. Rimmer J, Hellings P, Lund VJ, Alobid I, Beale T, Dassi C, et al. European position paper on diagnostic tools in rhinology. Rhinology. 2019 Jul 25;57(Suppl S28):1-41.

43. Bhattacharyya N. Ambulatory sinus and nasal surgery in the United States: demographics and perioperative outcomes. Laryngoscope. 2010 Mar;120(3):635-8.

44. Prokopakis EP, Koudounarakis El, Velegrakis GA. Efficacy of inferior turbinoplasty with the use of $\mathrm{CO}(2)$ laser, radiofrequency, and electrocautery. Am J Rhinol Allergy. 2014 
Jun;28(3):269-72

45. Bizakis JG, Lachanas VA, Drivas El, Kyrmizakis DE, Prokopakis EP, Benakis AA, et al. Cocaine flakes versus tetracaine/adrenaline solution for local anaesthesia in septoplasty. Rhinology. 2004 Dec;42(4):236-8.

46. Prokopakis EP, Vardouniotis AS, Karatzanis AD, Malandrakis SG, Siganos CS, Velegrakis GA. Long-term results of intranasal laserassisted dacryocystorhinostomy with the use of a surgical microscope. Auris Nasus Larynx. 2010 Feb;37(1):66-70.

47. DeConde AS, Mace JC, Levy JM, Rudmik L, Alt JA, Smith TL. Prevalence of polyp recurrence after endoscopic sinus surgery for chronic rhinosinusitis with nasal polyposis. Laryngoscope. 2017;127(3):550-5.

48. Philpott C, Hopkins C, Erskine S, Kumar N, Robertson A, Farboud A, et al. The burden of revision sinonasal surgery in the UK-data from the Chronic Rhinosinusitis Epidemiology Study (CRES): a crosssectional study. BMJ Open. 2015 Apr 29;5(4):e006680.

49. Rudmik L, Soler ZM, Hopkins C, Schlosser RJ, Peters A, White AA, et al. Defining appropriateness criteria for endoscopic sinus surgery during management of uncomplicated adult chronic rhinosinusitis: a RAND/ UCLA appropriateness study. Rhinology. 2016 Jun:54(2):117-28.

50. Calus L, Van Bruaene N, Bosteels C, Dejonckheere S, Van Zele T, Holtappels $G$, et al. Twelve-year follow-up study after endoscopic sinus surgery in patients with chronic rhinosinusitis with nasal polyposis. Clin Transl Allergy. 2019;9:30.

51. Alsharif $\mathrm{S}$, Jonstam $\mathrm{K}$, van Zele $\mathrm{T}$, Gevaert $\mathrm{P}$, Holtappels G, Bachert C. Endoscopic Sinus Surgery for Type-2 CRS wNP: An EndotypeBased Retrospective Study. Laryngoscope. 2019;129(6):1286-92

52. van der Veen J, Seys SF, Timmermans M Levie P, Jorissen M, Fokkens WJ, et al. Reallife study showing uncontrolled rhinosinusitis after sinus surgery in a tertiary referral centre. Allergy. 2017 Feb;72(2):282-90

53. Fajt ML, Wenzel SE. Development of New Therapies for Severe Asthma. Allergy Asthma Immunol Res. 2017 Jan:9(1):3-14.

54. Orlandi RR, Kingdom TT, Hwang PH, Smith TL, Alt JA, Baroody FM, et al. International
Consensus Statement on Allergy and Rhinology: Rhinosinusitis. Int Forum Allergy Rhinol. 2016 Feb;6 Suppl 1:S22-209.

55. Bachert C, Zhang N, Hellings PW Bousquet J. Endotype-driven care pathways in patients with chronic rhinosinusitis. J Allergy Clin Immunol. 2018 May;141(5):1543-51.

56. Khan A, Huynh TMT, Vandeplas G, Joish VN, Mannent LP, Tomassen $P$, et al. The GALEN rhinosinusitis cohort: chronic rhinosinusitis with nasal polyps affects healthrelated quality of life. Rhinology. 2019 Oct 1:57(5):343-51.

57. Bachert C, Han JK, Desrosiers M, Hellings PW, Amin N, Lee SE, et al. Efficacy and safety of dupilumab in patients with severe chronic rhinosinusitis with nasal polyps (LIBERTY NP SINUS-24 and LIBERTY NP SINUS-52): results from two multicentre, randomised double-blind, placebo-controlled, parallelgroup phase 3 trials. Lancet Lond Engl. 2019 Sep 19;

58. Tomassen P, Vandeplas $G$, Van Zele T, Cardell L-O, Arebro J, Olze H, et al. Inflammatory endotypes of chronic rhinosinusitis based on cluster analysis of biomarkers. J Allergy Clin Immunol. 2016;137(5):1449-1456.e4.

59. Kowalski ML, Agache I, Bavbek S, Bakirtas A, Blanca M, Bochenek G, et al. Diagnosis and management of NSAID-Exacerbated Respiratory Disease (N-ERD)-a EAACI position paper. Allergy. 2019;74(1):28-39.

60. Bachert C, Zhang L, Gevaert P. Current and future treatment options for adult chronic rhinosinusitis: Focus on nasal polyposis. J Allergy Clin Immunol. 2015 Dec;136(6):1431-40

61. Bachert C, Hellings PW, Mullol J, Hamilos DL, Gevaert P, Naclerio RM, et al. Dupilumab improves health-related quality of life in patients with chronic rhinosinusitis with nasal polyposis. Allergy. 2020 Jan;75(1):148157.

2. Bachert C, Zinreich SJ, Hellings PW, Mullol J, Hamilos DL, Gevaert P, et al. Dupilumab reduces opacification across all sinuses and related symptoms in patients with CRSwNP. Rhinology. 2020 Feb 1;58(1):10-17.

63. Hellings PW. EPOS2020 and beyond. Rhinology. 2019 Jun 1;57(3):161.

64. Fokkens WJ, Lund V, Bachert C, Mullol J,
Bjermer L, Bousquet J, et al. EUFOREA consensus on biologics for CRSWNP with or without asthma. Allergy. 2019 Dec;74(12):2312-2319.

65. Ren L, Zhang N, Zhang L, Bachert C. Biologics for the treatment of chronic rhinosinusitis with nasal polyps - state of the art. World Allergy Organ J. 2019 Aug;12(8):100050

66. Hopkins C, Williamson E, Morris S, Clarke CS, Thomas M, Evans H, et al. Antibiotic usage in chronic rhinosinusitis: analysis of national primary care electronic health records. Rhinology. 2019 Dec 1;57(6):420-429.

67. Fokkens WJ. Long-term antibiotics for Chronic Rhinosinusitis: changing views. Rhinology. 2019 Aug 1;57(4):241.

68. Karatzanis A, Chatzidakis A, Milioni A Vlaminck S, Kawauchi H, Velegrakis $S$, et al. Contemporary Use of Corticosteroids in Rhinology. Curr Allergy Asthma Rep. 2017 Feb;17(2):11.

69. Prokopakis E, Vlastos I, Pant H, Ferguson BJ. Chronic rhinosinusitis: observation or treatment. Curr Opin Allergy Clin Immunol. 2013 Feb;13(1):31-6.

70. Doulaptsi M, Prokopakis E, Seys S, Pugin B, Steelant B, Hellings P. Visual analogue scale for sino-nasal symptoms severity correlates with sino-nasal outcome test 22: paving the way for a simple outcome tool of CRS burden. Clin Transl Allergy. 2018;8:32.

Emmanuel Prokopakis, Professor Department of Otorhinolaryngology Head and Neck Surgery University of Crete School of Medicine,

Panepistimiou Avenue, 71110 Heraklion, Crete

Greece

Tel: +30 6932237622 ,

Fax: +30 2810542106

E-mail: eprokopakis@gmail.com 